\title{
Neutralité et politique de développement
}

\section{Catherine Schümperli Younossian}

\section{OpenEdition \\ Journals}

Édition électronique

URL : http://journals.openedition.org/aspd/199

DOI : 10.4000/aspd.199

ISSN : 1663-9669

\section{Éditeur}

Institut de hautes études internationales et du développement

\section{Édition imprimée}

Date de publication : 1 avril 2008

Pagination : XI-XVI

ISBN : 978-2-940415-01-4

ISSN : 1660-5934

Référence électronique

Catherine Schümperli Younossian, « Neutralité et politique de développement », Annuaire suisse de politique de développement [En ligne], 27-1 | 2008, mis en ligne le 30 juin 2009, consulté le 24 septembre 2020. URL : http://journals.openedition.org/aspd/199; DOI : https://doi.org/10.4000/aspd. 199 


\section{Introduction}

Neutralité et politique de développement

0 UELLE EST ENCORE la valeur de la «neutralité», mythe fondateur de l'identité nationale suisse? Le concept de la neutralité a fait l'objet de diverses interprétations au cours du $\mathrm{XX}^{\mathrm{e}}$ siècle et se trouve actuellement au centre du débat de la politique étrangère de la Suisse. Dans le courant de l'été 2007, le journal Le Temps consacre un dossier à la neutralité «qui revient curieusement à la mode aujourd'hui, alors que les menaces qui la justifiaient ont disparu $»^{1}$. L'enjeu actuel se joue entre deux lectures possibles: la neutralité «active» ou «intégrale». Mais c'est compter sans l'usage quelque peu abusif du terme de «neutralité climatique» utilisé en marge du débat sur le changement climatique. Arrêtons-nous sur quelques temps forts qui ont marqué le débat relatif à la neutralité en 2007, plus largement détaillés dans certains chapitres du présent Annuaire.

\section{La «neutralité climatique»}

Surprenante est la référence au principe de «neutralité climatique» proposée par le Secrétariat d'Etat à l'économie (SECO), qui «entend par là le fait d'engager tous les émetteurs de gaz à effet de serre en Suisse à prendre des mesures de compensation à l'étranger à hauteur de leurs rejets $»^{2}$. Moyen «habile» pour la Suisse de s'engager à réduire ses émissions de $100 \%$... sans avoir à se fixer d'objectifs contraignants! Les réactions ont été vives et sans nuance: plébiscitée par les milieux économiques qui y voient un moyen d'éviter des mesures par trop restrictives, les organisations non gouvernementales d'environnement et de développement ont vivement réagi à «cette fausse bonne idée», indéfendable en termes de politique de développement. Le principe de «neutralité climatique» n'est pas universel, puisqu'il permet à certains Etats seulement d'éviter de se soumettre à une réduction effective des émissions de $\mathrm{CO}_{2}$; le marché des crédits du carbone est inéquitable, car les pays pauvres n'en profitent guère, ne produisant que très peu de $\mathrm{CO}_{2}$ par habitant; enfin, il permet à la Suisse de s'acquitter à bon compte, voire de les contourner habilement, des obligations découlant du principe du «pollueur payeur» et de la «responsabilité commune mais différenciée ${ }^{3}$ face au réchauffement climatique. (Chapitre 10, «Environnement et développement»).

Evinçons donc d'emblée cette utilisation à mauvais escient du mot «neutralité» pour nous pencher sur une définition plus classique de la neutralité dans le contexte de la politique étrangère et de sécurité de la Suisse. Au sens du droit

1 «Que vaut encore la neutralité ?», Le Temps, 3 juillet 2007. A l'occasion de la parution de l'ouvrage de J.-J. Langendorf, Histoire de la neutralité. Une perspective, Gollion, Infolio, 2007.

2 T. Roth, «La neutralité climatique: une option pour la Suisse après 2012 ? », La Vie économique, n 9, 2007.

3 M. Egger, «En matière de climat, "neutralité" ne rime pas forcément avec "responsabilité"», Le Temps, 14 décembre 2007. 
international, «neutralité» signifie «non-participation militaire d'un Etat à un conflit armé opposant d'autres Etats $»^{4}$. Attachons-nous aux différentes interprétations qui ont cours dans le débat public actuel où deux visions s'affrontent, la neutralité «active», promue par Micheline Calmy-Rey, conseillère fédérale en charge des Affaires étrangères, et la neutralité «intégrale», défendue par le mouvement conservateur ASIN (Action pour une Suisse indépendante et neutre).

\section{Neutralité « active " versus neutralité « intégrale »}

Le concept de la neutralité est au cœur de la politique étrangère menée par la conseillère fédérale Micheline Calmy-Rey; il est l'instrument auquel elle souhaite donner un nouvel élan. Plaidant pour une neutralité dite active, la conseillère fédérale cherche à lever un tabou en invoquant la possibilité pour la Suisse de revendiquer un siège au sein du Conseil de sécurité de l'ONU. Le Rapport de politique étrangère 2007 offre à cet égard une argumentation détaillée, arguant du fait que la participation de la Suisse aux travaux du Conseil de sécurité soulignerait l'importance que la Suisse accorde à la transparence et à la légitimité de cet organe. Il est également relevé que d'autres Etats neutres, au rang desquels l'Autriche, la Suède, la Finlande et l'Irlande, y ont occupé un siège ${ }^{5}$.

La réaction de l'ASIN couvait depuis quelque temps et a été rendue publique sous la forme d'un argumentaire qui s'interroge sur la pertinence de renoncer à la neutralité suisse, «cet instrument qui a assuré jusqu'ici notre sécurité et qui nous a permis de pratiquer avec succès notre diplomatie de paix et notre aide humanitaire?». Dans son «livre noir» consacré à la neutralitéf, l'ASIN oppose son interprétation de la «neutralité intégrale» à la «neutralité active», terme qualifié de «moderniste et idiot» et dont elle préconise l'abolition. Elle dresse la liste des «infractions» à la neutralité commises, à ses yeux, tant par le Département des affaires étrangères (DFAE) que par celui de la Défense, de la Protection de la population et des Sports (DDPS), ainsi que par le Parlement. (Chapitre 1, «Politique extérieure»).

\section{La neutralité et la politique de paix et de sécurité}

Les notions de paix et de sécurité «se sont invitées à la table» de la coopération internationale, obligeant également la coopération suisse à mieux tenir compte des situations de conflits, latents ou ouverts, dans ses pays d'intervention ${ }^{7}$. Par l'adoption, en 1999, du rapport du Conseil fédéral sur la politique de sécurité, un pas de plus est franchi. Ce texte étend la notion de sécurité aux menaces non militaires et, surtout, ouvre la possibilité de principe pour des soldats suisses de participer à la coopération en matière de politique de sécurité en

4 Département fédéral des Affaires étrangères (DFAE), L'essentiel sur la neutralité suisse, s.d., $<$ http://www.eda.admin.ch > > thèmes >droit international public >droit international général >la neutralité de la Suisse.

5 Conseil fédéral, Neutralité (FF 2007 5283), annexe 1 du Rapport de politique étrangère, juin $2007 \mathrm{du}$ 15 juin 2007 (FF 2007 5257).

ASIN, Livre noir de la neutralité suisse, situation : 10 janvier 2008, <http://www.auns.ch>.

Paix et sécurité: les défis lancés à la coopération internationale, dossier de l'Annuaire suisse de politique de développement, Genève, Institut universitaire d'études du développement, 2006. 
Europe $^{8}$. Dans ce contexte, il convient de noter la participation de la Suisse au Partenariat pour la paix depuis 1996 et l'engagement d'unités de l'armée suisse dans des missions internationales de paix sous mandat de l'ONU (BosnieHerzégovine depuis 1996, Kosovo depuis 1999, Afghanistan depuis 2003). Les principes de neutralité et de solidarité y sont redéfinis en fonction de la nouvelle réalité internationale. (Chapitre 11, «Politique de paix et politique de sécurité».)

«Sans sécurité, pas de développement»: aussi l'indice de l'engagement pour le développement (CDI, Commitment to Development Index $)^{9}$, qui vise à mesurer l'engagement des pays industrialisés en faveur du développement, accorde-t-il une place importante à la sécurité. En 2007, la Suisse a été rétrogradée du $13^{\mathrm{e}}$ au $17^{\mathrm{e}}$ rang. Ce mauvais résultat s'explique notamment par sa très faible contribution aux opérations internationales de maintien de la paix menées par l'ONU. (Chapitre 2, «Coopération avec les pays en développement».)

\section{La neutralité et la coopération multilatérale au développement}

Le principe de neutralité sert également d'autres intérêts que celui de la sécurité nationale. La coopération multilatérale, volet important de la coopération au développement, va trouver un nouvel élan dans les années 1990 à la suite de l'adhésion de la Suisse aux principales organisations internationales de développement; en 1992, le peuple accepte en votation populaire l'adhésion de la Suisse au Fonds monétaire international et à la Banque mondiale, avec 55,8\% des voix, puis en 2002 l'adhésion à l'Organisation des Nations unies, avec $54,6 \%$ des voix. Il aura fallu près d'un demi-siècle pour qu'une petite majorité du peuple suisse ne voie pas de contradiction majeure entre le principe de neutralité et une participation politique, pleine et entière, dans les organisations internationales - et cette participation continue d'être combattue par une partie de l'opinion publique suisse et par l'ASIN, au nom de la neutralité ${ }^{10}$.

La neutralité soumise au débat public en 2008

Durant le $\mathrm{XX}^{\mathrm{e}}$ siècle, la politique de neutralité a pris différentes facettes. Durant la Seconde Guerre mondiale et pendant le conflit Est-Ouest, la Suisse a mené une politique de neutralité dite «intégrale», caractérisée par aucune immixtion, qu'elle soit militaire, économique ou politique, dans les conflits survenant entre d'autres Etats ${ }^{11}$. Après la disparition de l'ordre mondial bipolaire, la Suisse s'est

8 La sécurité par la coopération. Rapport du Conseil fédéral à l'Assemblée fédérale sur la politique de sécurité de la Suisse (RAPOLSEC 2000) du 7 juin 1999 (FF 1999 6903).

9 L'indice de l'engagement pour le développement (CDI - Commitment to Development Index) permet de classer 21 des pays les plus riches en fonction de l'engagement de ces derniers à l'égard des politiques en faveur des pays pauvres. Allant au-delà des comparaisons de base sur l'aide publique au développement (APD), le CDI mesure les efforts des pays selon sept critères importants pour les pays en développement: l'aide internationale, le commerce, les investissements, la politique migratoire, l'environnement, la sécurité et la technologie (voir <http://www.cgdev.org/cdi>).

10 C. Schümperli, La politique suisse de solidarité internationale. De la coopération au développement global, coll. Le Savoir suisse, Lausanne, Presses Polytechniques et Universitaires Romandes (PPUR), 2007.

11 L. Goetschel, M. Bernath et D. Schwarz, Politique extérieure suisse. Fondements et possibilités, coll. Hinc \& Nunc, Lausanne, Editions Payot, 2004. 
trouvée confrontée à une nouvelle situation. Comme tous les Etats neutres, elle s'est déclarée prête à soutenir, en signe de solidarité, les mesures de contrainte, non militaires, prises par la communauté internationale en vue de maintenir ou de rétablir la paix. Depuis 1990, elle participe régulièrement aux sanctions internationales. Comment la neutralité sera-t-elle perçue et interprétée dans les années à venir? La question sera au cœur des débats de politique étrangère, qui s'annoncent vifs et riches en polémiques.

Conscient de l'importance de la perception de l'opinion suisse, le DFAE s'est lancé dans une vaste campagne d'information auprès des citoyens sur six «grands aspects» de sa politique extérieure, la neutralité étant le premier point évoqué ${ }^{12}$. Dans une approche originale et novatrice - bien qu'on puisse douter de son impact et de la valeur représentative du sondage -, les citoyens sont invités à se prononcer sur les principales orientations de la politique extérieure en retournant au DFAE un questionnaire-sondage. Quelque huit mois après le lancement de cette campagne, plus de 1000 réponses ont été enregistrées. On y apprend notamment que $63 \%$ des personnes considèrent la neutralité comme très importante. Si un tiers des réponses soulignent que la pratique de la neutralité en l'état est trop défensive, elle est jugée satisfaisante par la moitié des réponses ${ }^{13}$. De son côté, l'ASIN lance une initiative populaire «Pour le renforcement des droits populaires dans la politique étrangère (accords internationaux: la parole au peuple !) », déclarée valide par la Chancellerie fédérale en février $2008^{14}$. L'ASIN aura jusqu'en septembre 2009 pour récolter les 100000 signatures requises.

La neutralité est sans conteste un élément identitaire fort de la politique extérieure de la Suisse. Si ce concept a évolué à travers l'histoire récente du pays et a su s'adapter aux nouvelles réalités internationales, il faut rappeler son objectif premier: «La neutralité sert la sécurité nationale, mais c'est aussi un message de paix: un pays neutre cherche avant tout à éviter la guerre. ${ }^{15} \mathrm{La}$ marche du monde et le positionnement de la Suisse sur le plan international imposent une réinterprétation constante de la neutralité. Pour lui permettre d'évoluer dans un climat politique interne où les forces conservatrices plaident pour une interprétation restrictive, il est important d'en définir clairement les champs d'application.

Au début de ce troisième millénaire, les changements climatiques sont incontestablement la menace la plus forte pour l'équilibre planétaire; les enjeux sont trop graves et globaux pour qu' on joue avec une prétendue «neutralité climatique». Par ailleurs, de grands débats sont à l'ordre du jour de l'agenda de la coopération internationale en 2008: la question de l'efficacité de l'aide sera analysée à Accra en septembre, et celle du financement du développement sera débattue à Doha en novembre. Dans ce contexte, c'est à son engagement solidaire, bien plus qu'à sa neutralité, que la contribution de la Suisse sera mesurée !

$* * *$

12 DFAE, Une politique extérieure du dialogue, Berne, s.d.

13 DFAE, <http://www.eda.admin.ch> >documentation > publications > une politique extérieure du dialogue (consultation : février 2008).

14 FF 20081333.

15 DFAE, Une politique extérieure du dialogue, op. cit., p. 7. 
Les migrations internationales sont devenues un enjeu primordial à l'échelle de la planète, et désormais indissociable des questions liées au développement. Avec le lancement de l'Initiative de Berne en 2001, la Suisse a donné une impulsion importante pour le renforcement de la coopération internationale dans le domaine migratoire, qui a débouché sur la création de la Commission mondiale sur les migrations internationales.

Au niveau mondial, on compte environ 200 millions de migrants, dont près de la moitié sont des femmes. En Suisse, un habitant sur trois a des origines étrangères et un quart de la population active ne possède pas de passeport suisse. Face à la complexification des flux migratoires, les approches unilatérales pour réguler les migrations apparaissent de plus en plus inopérantes. Même si les Etats restent très jaloux de leur souveraineté en la matière, ils prennent peu à peu conscience du fait que seule une action concertée au niveau international est à même d'aboutir à des solutions constructives, acceptables à la fois pour les pays d'origine, de transit et de destination - qui tendent parfois à se confondre et pour les migrants et migrantes eux-mêmes.

Conjointement à ces initiatives politiques, qui ont elles-mêmes donné lieu à de nombreuses consultations régionales et nationales, les études menées sur ce qu'on désigne en anglais par le migration-development nexus se multiplient, même si elles restent encore largement insuffisantes pour éclairer toutes les facettes du lien entre les deux phénomènes. D'une part, on prend mieux en compte les conséquences des mouvements migratoires sur les pays d'origine et d'accueil et, d'autre part, on cerne, dans toute sa complexité, l'impact du développement sur les migrations Sud-Nord, discuté longtemps de manière très controversée. Si l'aide humanitaire peut, dans l'immédiat, prévenir l'émigration forcée, en particulier régionale, le développement économique renforce parfois la pression migratoire à moyen terme avant de contribuer à sa diminution à plus long terme (migration hump). Ainsi, les visions de la coopération au développement et de la politique migratoire ont pu paraître antagonistes par le passé et continuent de l'être dans une perspective focalisée sur l'avenir immédiat. En revanche, ces optiques divergentes sont susceptibles de s'accorder si l'on adopte une approche de développement durable. Ces différentes visions seront analysées et discutées dans le prochain dossier de l'Annuaire (parution en hiver 2008-2009) ${ }^{16}$.

$$
* * *
$$

Dès cette année, l'Annuaire suisse de politique de développement est publié par l'Institut de hautes études internationales et du développement, issu du regrou-

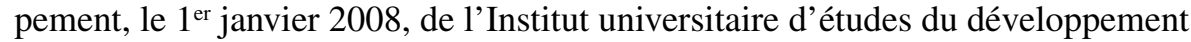
(IUED), anciennement éditeur, et de l'Institut universitaire de hautes études internationales (HEI).

Autre nouveauté, dès le $1^{\text {er }}$ juin 2008 , la totalité des textes publiés dans l'Annuaire ces cinq dernières années (2002-2007) sera mise en ligne en libre accès. Une plus grande complémentarité sera opérée entre la version papier et le site

16 D. Efionayi, extrait du synopsis du dossier «Migration et développement», octobre 2007. 
Internet. A titre d'exemple, des données statistiques additionnelles seront offertes sur nos pages Internet ${ }^{17}$.

Le comité de rédaction tient à remercier vivement les auteurs des textes publiés dans ce nouveau volume de l'Annuaire suisse de politique de développement, ainsi que les nombreux spécialistes de l'Administration fédérale, des ONG de développement et des milieux académiques qui, par leur relecture critique ou leurs précieux conseils, assurent une grande rigueur à notre publication. Notre gratitude va également à celles et ceux qui contribuent à la conception, à la réalisation et à la traduction de cette publication. C'est grâce à la compétence et à l'engagement de toutes et tous que la fiabilité de cet ouvrage de référence est garantie. Nous remercions également la Direction du développement et de la coopération (DDC) pour son appui financier.

Pour conclure, le comité de rédaction tient à rendre un chaleureux hommage à Jacques Vallet, secrétaire de rédaction de l'Annuaire depuis son lancement, et à Xavier Tschumi Canosa, rédacteur de l'Annuaire. Pendant près de trente ans, Jacques Vallet s'est engagé, avec grande compétence et dévouement, à la publication des nombreux volumes de la collection. Participant à près de 300 séances de comité, il a accompagné le processus de la conception à la phase finale d'impression, tout en lui assurant une large diffusion. Arrivé à l'âge de la retraite, Jacques Vallet a quitté ses fonctions au début de janvier 2008. Sa personnalité humaine et chaleureuse manquera à toute l'équipe de l'Annuaire. Xavier Tschumi Canosa a collaboré pendant près de six ans à la rédaction des textes de l'Annuaire et a dirigé le volume consacré à la politique de paix et de sécurité (dossier 2006). Précision et rigueur ont caractérisé son travail, chaleur humaine et amitié ses relations avec ses collègues. Passionné par les questions environnementales, Xavier Tschumi Canosa poursuivra sa carrière comme collaborateur scientifique au sein de l'Office fédéral de l'environnement. Le comité de rédaction les remercie tous deux vivement pour ces années d'engagement et leur adresse ses vœux les plus sincères pour cette nouvelle étape de vie.

Catherine Schümperli Younossian Directrice de la rédaction

17 Voir la page du site de l'Institut de hautes études internationales et du développement consacrée à l'Annuaire: <http://graduateinstitute.ch/development/publications/aspd.html>. 